\title{
Predicción in vitro del estrés de Vallisneria americana Michx. por enriquecimiento de iones amonio y nitrato
}

\section{In vitro prediction of Vallisneria americana Michx. stress by enrichment of ammonium and nitrate ions}

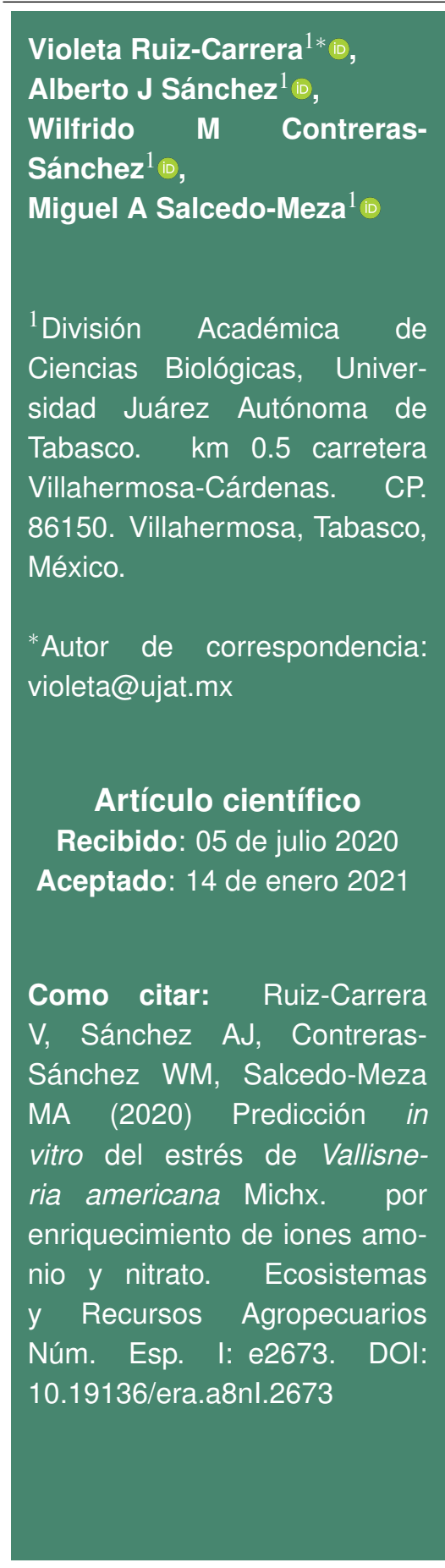

RESUMEN. El enriquecimiento de nutrientes es un factor de estrés involucrado en la declinación de vegetación sumergida. Sin embargo, la sensibilidad fisiológica o toxicológica de los genotipos varía debido a las interacciones microbióticas y el desbalance fisicoquímico del agua, las cuales han sido abordadas mediante una aproximación reduccionista y experimentos controlados. El objetivo del estudio fue evaluar, en un modelo de cultivo in vitro de Vallisneria americana, diferentes fuentes y concentraciones de $\mathrm{N}$ sobre la supervivencia y el crecimiento de hojas y raíces. Las plantas juveniles in vitro se expusieron a concentraciones de 500 a $2000 \mu \mathrm{g} \mathrm{L}^{-1}$ de $\mathrm{N}$ total con fuentes de $\mathrm{NH}_{4}, \mathrm{NO}_{3}$ y $\mathrm{NH}_{4}: \mathrm{NO}_{3}$ (1:1) en un diseño factorial $4 \times 3$, en evaluaciones de corto y mediano plazo. La supervivencia fue inversamente proporcional a los síntomas de clorosis, necrosis o ruptura de tejidos. La primera elongación foliar se midió con un método no destructivo. Mientras que a los 25 días, se cuantificó la longitud individual y promedio de hojas y raíces, número de hojas regeneradas y número de raíces. Las plantas no mostraron síntomas de vulnerabilidad. El $\mathrm{NH}_{4}$ en todas sus concentraciones inhibió el crecimiento de los nueve parámetros analizados. En contraste, el $\mathrm{NH}_{4}: \mathrm{NO}_{3}$ causó dos inhibiciones a nivel de hoja y el $\mathrm{NO}_{3}$ estimuló todos los crecimientos de hojas y raíces. La predicción de crecimiento resulta prometedora para discriminar factores de estrés sobre la vegetación sumergida de los Pantanos de Centla.

Palabras clave: Cintilla, estrés in vitro, inhibición fisiológica, macrófita sumergida, $\mathrm{N}$ inorgánico.

ABSTRACT. Nutrient enrichment is an environmental stress factor involved in the decline of submerged vegetation. However, the physiological or toxicological sensitivity of the genotypes varies due to microbiotic interactions and physicochemical imbalance of water, which have been studied through a reductionist approach and controlled experiments. The objective of the study was to evaluate, in an in vitro culture model of Vallisneria americana, the increase of different sources and concentrations of $\mathrm{N}$ on the survival and growth of leaves and roots. Juvenile plants in vitro grew at concentrations of 500 to $2000 \mu \mathrm{g} \mathrm{L}^{-1}$ of total $\mathrm{N}$ with sources of $\mathrm{NH}_{4}, \mathrm{NO}_{3}$ and $\mathrm{NH}_{4}: \mathrm{NO}_{3}(1: 1)$ in a factorial design $4 \times 3$, in short- and medium-term evaluations. Survival was inversely proportional to symptoms of chlorosis, necrosis, or tissue rupture. The first foliar elongation was measured with a non-destructive method. While at 25 days, the individual and average length of leaves and roots, number of regenerated leaves and number of total roots were quantified. The plants did not show symptoms of vulnerability. $\mathrm{NH}_{4}$ in all its concentrations inhibited the growth of the nine parameters analyzed. In contrast, $\mathrm{NH}_{4}: \mathrm{NO}_{3}$ caused two inhibitions at the leaf level and $\mathrm{NO}_{3}$ stimulated leaf and root growth. The growth prediction proved promising to discriminate stress factors on the submerged vegetation of the Pantanos de Centla.

Key words: Watercelery, in vitro stress, physiological inhibition, submerged macrophyte, inorganic $\mathrm{N}$. 


\section{INTRODUCCIÓN}

La mayoría de los humedales fluviales en las planicies costeras del mundo registran alta concentración de nutrientes relacionadas con la eutrofización que provoca declinación o pérdida de macrófitas sumergidas enraizadas (Salk et al. 2018, CruzRamírez et al. 2019). La explicación de los efectos de la eutrofización sobre esta vegetación se complica por estresores aditivos como salinidad, nutrientes, $\mathrm{pH}$, oxígeno disuelto y luz, incluidos los parámetros reguladores como perifiton, fitoplancton, sólidos totales suspendidos y concentración de clorofila, y más reciente el incremento de $\mathrm{CO}_{2}$ atmosférico que eleva la temperatura en los ecosistemas acuáticos (Moreno-Marín et al. 2018, Li et al. 2019, Tootoonchi et al. 2020). La drástica reducción de biomasa sumergida impacta en la provisión de alimento y de hábitat estructurados, lo que favorece la riqueza y dominancia de los productores primarios, así como los procesos biogeoquímicos asociados que influyen en la asimilación de nutrientes, la mejora en la calidad del agua y el incremento de fauna acuática (Yang et al. 2008, Kouamé et al. 2011, Lone et al. 2014). En consecuencia, el fenómeno de eutrofización altera el ciclo del nitrógeno y los procesos ecológicos de los sistemas acuáticos que benefician la dinámica de las especies nativas y la economía local, lo cual causa daños irreversibles a la calidad ambiental con los riesgos potenciales para la salud humana (Salcedo et al. 2012, Sánchez et al. 2012, Cruz-Ramírez et al. 2019). Adicionalmente, en la literatura prevalece la hipótesis que la pérdida de vegetación sumergida ha ocurrido por el enriquecimiento de N (Naiman y Dudgeon 2011, Le Moal et al. 2019). Este enriquecimiento ha sido principalmente asociado con los escurrimientos de áreas agropecuarias y la descarga de aguas residuales municipales con deficiente o nulo tratamiento (Cordovil et al. 2018).

La supervivencia y el crecimiento de macrófitas sumergidas enraizadas en experimentos de enriquecimiento de $\mathrm{N}$ en mesocosmos y campo presenta diferentes tipos y magnitudes de estrés por el estado redox de los iones $\mathrm{N}$ y las interferencias de factores bióticos y abióticos implicados (Leoni et al. 2008, Zaman y Asaeda 2013, Gao et al. 2019). Las plantas adultas de Vallisneria natans (Lour.) H.Hara manifestaron estrés fisiológico en mesocosmos y estrés tóxico en campo en concentración moderada de $\mathrm{N}$ usando soluciones de $\mathrm{NH}_{4} \mathrm{Cl}$ y $\mathrm{NaNO}_{3}$, respectivamente (Cao et al. 2007, 2009). En contraste, V. spinulosa S. Z. Yan superó el estrés tóxico con suministro de $\mathrm{NH}_{4} \mathrm{NO}_{3}$ pero el crecimiento vegetativo fue inhibido (Zhao et al. 2016). Una alternativa experimental para sustentar hipótesis ambientales son las predicciones reduccionistas a escala in vitro (Ruiz-Carrera y Sánchez 2008, Teh et al. 2014, Bautista-Regil et al. 2016). Principalmente en la etapa juvenil, cuando decae el éxito reproductivo en campo (McFarland 2006) y la repoblación con germinados es poco exitosa (Schloesser y Manny 2007, Johnson et al. 2019) es imperativo definir el mecanismo fisiológico por el cual el enriquecimiento de $\mathrm{N}$ afecta el estado ecológico de las poblaciones de $V$. americana Michx. La condición aséptica inherente al cultivo in vitro favorece la exclusión de microorganismos que reducen $\mathrm{NO}_{3}$ y oxidan $\mathrm{NH}_{4}$ en el ambiente (Padgett y Leonard 1993), reducen los efectos individuales y combinados de los factores ambientales o nutricionales previamente señalados como causantes de la declinación de vegetación sumergida, y evitan la herbivoría involucrada en la pérdida de su potencial reproductivo (Balestri y Cinelli 2003).

En las poblaciones vegetales en proceso de declinación crítica con amplias variaciones locales y baja persistencia localizadas en Áreas Naturales Protegidas, como en la Reserva de la Biosfera Pantanos de Centla (Sánchez et al. 2012), un problema a resolver es determinar el impacto directo del enriquecimiento de $\mathrm{N}$ con especies iónicas de $\mathrm{NH}_{4}$ y $\mathrm{NO}_{3}$ en la supervivencia o el crecimiento de $V$. americana de Pantanos de Centla. El objetivo de este estudio fue discriminar los efectos del enriquecimiento de las diferentes fuentes iónicas de $\mathrm{N}$ sobre la sobrevivencia de germinados y las respuestas de crecimiento de hojas y raíces mediante el uso de un modelo de cultivo in vitro de del ecotipo de V. americana (Ruiz-Carrera y Sánchez 2008). En 
esta escala reduccionista, la hipótesis fue que el crecimiento de hojas y raíces de $V$. americana disminuye con incrementos de $\mathrm{NH}_{4}$ y el co-suministro de $\mathrm{NH}_{4}: \mathrm{NO}_{3}$, pero sin afectar la sobrevivencia. La hipótesis se resolvió mediante el registro de la supervivencia y los marcadores de elongación foliar, longitud individual y promedio de hojas y raíces, número de hojas regeneradas y número de raíces totales en dos evaluaciones experimentales in vitro, de corto y mediano plazo.

\section{MATERIALES Y MÉTODOS}

\section{Ecotipo y preparación de semillas}

La angiosperma sumergida enraizada V. americana (cintilla silvestre) de la familia Hydrocharitaceae es dominante en la Reserva de la Biosfera Pantanos de Centla ( $17^{\circ} 57^{\prime} 53^{\prime \prime}$ y $18^{\circ} 39^{\prime} 03^{\prime \prime}$ N, $92^{\circ} 06^{\prime} 39^{\prime \prime}$ y $92^{\circ} 47^{\prime} 58^{\prime \prime}$ O), que está localizada en la cuenca baja de los ríos Usumacinta y Grijalva. Las poblaciones de $V$. americana han registrado amplias variaciones en su abundancia y área de los parches, que han implicado su desaparición temporal en algunos sitios (Sánchez et al. 2012).

Los frutos de $V$. americana fueron recolectados en la Laguna de San Pedrito $\left(54^{\circ} 25^{\prime} 50^{\prime \prime} \mathrm{N}-20^{\circ}\right.$ 30'63.2" O) en la Reserva de la Biosfera Pantanos de Centla, para luego transportarlos al laboratorio a temperatura ambiente y sumergidos en agua de la laguna. La superficie longitudinal del fruto se cortó con bisturí para extraer las semillas y conservarlas en refrigeración a temperatura de $12 \pm 2{ }^{\circ} \mathrm{C}$ con el mucílago acompañante y con recambio diario de agua de la llave durante una semana.

\section{Regeneración in vitro}

Las plantas in vitro de $V$. americana se regeneraron de semillas asépticas en medio de cultivo de doble fase (columna de agua y soporte) $\sin \mathrm{N}$ (Ruiz-Carrera y Sánchez 2008). En la asepsia de las semillas se usó $0.6 \%$ de $\mathrm{NaClO}$ durante $10 \mathrm{~min}$ y tres lavados de agua estéril. La fase de soporte y la fase acuosa presentaron una relación de 3.6:1 $\mathrm{cm}$ de altura. El soporte fue preparado con $4 \%$ de agar-agar y se esterilizó en tubo de ensayo (TE $25 \mathrm{x}$
$150 \mathrm{~mm}$ ) cubierto de papel aluminio. Un mililitro de la fase acuosa estéril se agregó al tubo con soporte de agar por medio de una jeringa desechable estéril. Máximo siete semillas asépticas fueron sembradas en la unidad bifásica de cultivo. El número de semillas fue variable por el inconveniente del tamaño pequeño, su elevada adherencia y para evitar su deshidratación durante transferencias prolongadas. Las plantas se regeneraron a $30 \pm 5^{\circ} \mathrm{C}$ en un cuarto de crecimiento con $16 \mathrm{~h}$ de iluminación tenue (flujo de fotones de $1 \mathrm{~mol} \mathrm{~m}^{-2} \mathrm{~s}^{-1}$ ) y $8 \mathrm{~h}$ de oscuridad. En esta condición las plántulas desarrollaron raíces, lo que indicó en operación la fase de crecimiento autotrófico. El volumen original del medio acuoso fue sustituido y duplicado por medio acuoso nitrogenado cuando la primera hoja del brote alcanzó la interfase agua-aire. La fase acuosa se renovó usando los procedimientos de la condición de regeneración. Las soluciones con $\mathrm{N}$ se prepararon de estándares de $\mathrm{NH}_{4} \mathrm{Cl}$ y $\mathrm{NaNO}_{3}$ de $1 \mathrm{mg} \mathrm{mL}^{-1}$. En la preparación de las dos fases y los estándares se utilizó agua dulce semidura reconstituida en agua desionizada cumpliendo con los parámetros de $\mathrm{pH}$, conductividad y alcalinidad y de sales inorgánicas recomendados para bioensayos de organismos acuáticos (Eaton et al. 2000). El intervalo de $\mathrm{pH}$ inicial y final de cada una de las diferentes fases con $\mathrm{N}$ fueron de 7.2-7.8 y 7.8-8.4, sin registrar diferencias significativas entre tratamientos $(p>$ 0.05). La esterilización de materiales, solución de estándares y fases se realizó en autoclave a $121^{\circ} \mathrm{C}$ y $104 \mathrm{kPa}$ por $15 \mathrm{~min}$.

\section{Evaluaciones de estrés}

Las unidades de cultivo con plantas in vitro regeneradas en medio bifásico libre de $\mathrm{N}$ que mostraron adecuados desarrollos de hojas y raíces se seleccionaron para realizar las evaluaciones de enriquecimiento de $\mathrm{N}$ de corto y mediano plazo. Los efectos individuales e interactivos de la concentración de $\mathrm{N}$ y la fuente de $\mathrm{N}$ se analizaron en un experimento factorial aleatorizado $(4 \times 3)$. En la concentración de $\mathrm{N}$ se usaron niveles de 500, 1000,1500 y $2000 \mu \mathrm{g}$ $\mathrm{L}^{-1} \mathrm{~N}$ Total (NT). Las fuentes de $\mathrm{N}$ fueron $\mathrm{NH}_{4}, \mathrm{NO}_{3}$ y el co-suministro 1:1 de $\mathrm{NH}_{4}: \mathrm{NO}_{3}$. El estrés tóxico se evaluó con síntomas que disminuyen la superviven- 
cia, como la clorosis foliar, necrosis o ruptura de tejidos. El efecto de estrés fisiológico se estimó en la planta completa con cinco marcadores de crecimiento de hojas y cuatro de raíces.

A corto plazo se registró la elongación de la primera hoja emergida de cada individuo a 2, 5 y 8 días de cultivo, usando un método no destructivo, que consistió en marcar sobre la superficie del tubo la posición inicial y final de la hoja erguida de cada individuo usando diferentes colores hasta la interfase agua - aire de la columna de agua con $\mathrm{N}$. La medición de elongación foliar se realizó con un vernier (precisión $0.05 \mathrm{~mm}$ ). Las hojas curvadas durante el transcurso del experimento se descartaron, lo cual cambió el diseño experimental a un arreglo desbalanceado. Las mediciones de elongación foliar variaron de 4 a 13 por tratamiento en mínimo tres repeticiones de la unidad de cultivo. El bioensayo de mediano plazo inició a los 10 días de la primera evaluación y transcurridos 25 días se extrajo la planta completa, se colocó sobre una caja de Petri y el gel adherido a las raíces se separó de forma manual. Los parámetros de crecimiento registrados en las hojas (excluido el coleóptilo) fueron la longitud de la primera hoja $\left(\mathrm{LH}_{1}\right)$, longitud de la segunda hoja $\left(\mathrm{LH}_{2}\right)$, longitud foliar promedio ( $\mathrm{LF}=\mathrm{H}_{1}+\mathrm{H}_{2} / 2$ ) y el número de nuevas hojas $\mathrm{NH}=\left(\mathrm{H}_{2}+\mathrm{H}_{3}+\mathrm{H}_{n}\right)$. Mientras que las mediciones en las raíces fueron la longitud de la primera raíz $\left(\mathrm{LR}_{1}\right)$, longitud de la segunda raíz $\left(\mathrm{LR}_{2}\right)$, longitud promedio de raíces ( $\left.L R=R_{1}+R_{2} / 2\right)$, y el número total de raíces $\left(T R=\mathrm{R}_{1}+\mathrm{R}_{2}+\mathrm{R}_{n}\right)$. Durante las dos evaluaciones experimentales, los cultivos permanecieron en un cuarto de crecimiento con $20 \mu \mathrm{mol}$ de fotones $\mathrm{m}^{-2} \mathrm{~s}^{-1}$, sin modificar el resto de los parámetros ambientales usados en la germinación.

\section{Análisis estadístico}

En la elongación foliar se aplicó un ANOVA factorial para estimar los efectos de la fuente y concentración de N, y un ANOVA simple para el tiempo; mientras que los cambios en longitud y número de estructuras vegetales se determinaron con ANOVA - MANCOVA usando como covariable el número de germinados por unidad experimental (G). La prueba de normalidad de Kolmorogov-Smirnnov y la homo- geneidad de varianzas de Cochran $\mathrm{C}$ se aplicaron a las variables y covariable. Con base en los resultados se usó la prueba de Kruskal-Wallis $(H)$ para la $\mathrm{LH}_{2}$ y las comparaciones post-hoc para la prueba de Fisher LSD $(p<0.05)$. Todos los datos se analizaron con el procedimiento de MGL del software Statistica (Statsoft 2007).

\section{RESULTADOS}

Las plantas juveniles sometidas a enriquecimiento de $\mathrm{N}$ en la columna de agua en concentraciones de 500 a $2000 \mu \mathrm{g} \mathrm{L}^{-1}$ NT y fuentes de $\mathrm{NH}_{4}, \mathrm{NO}_{3}$ y $\mathrm{NH}_{4}: \mathrm{NO}_{3}$ crecieron in vitro mostrando fenotipos normales, por lo que se descartó el estrés tóxico visible. Los efectos significativos $(p=$ 0.0001 ) se registraron para la elongación foliar por la fuente iónica de $\mathrm{N}$, la concentración de $\mathrm{N}$ y su interacción (Tabla 1, Figura 1). La elongación de la primera hoja presentó un promedio bajo con $\mathrm{NH}_{4}$ y alto con $\mathrm{NH}_{4}: \mathrm{NO}_{3}$ y NO 3 ( $<$ < 0.05). Mientras que, la interacción provocó elongaciones menores $(p<$ $0.05)$ en tres concentraciones de $\mathrm{NH}_{4}(500,1500 \mathrm{y}$ $\left.2000 \mu \mathrm{g} \mathrm{L}^{-1}\right)$, una de $\mathrm{NO}_{3}\left(500 \mu \mathrm{g} \mathrm{L}^{-1}\right)$ y dos de $\mathrm{NH}_{4}: \mathrm{NO}_{3}$ (500 y $1000 \mu \mathrm{g} \mathrm{L}^{-1}$ ). La elongación foliar fue significativa en el curso del tiempo $(p=0.0001)$ y mantuvo ascenso constante con las tres fuentes de $N(p<0.05)$.

Tabla 1. Variación en la elongación foliar de Vallisneria americana. FN = fuente de $\mathrm{N}, \mathrm{CN}=$ concentración de $\mathrm{N}, \mathrm{T}=$ tiempo de cultivo. ${ }^{a}=\mathrm{p}<$ $0.0001)$.

\begin{tabular}{lcccccc}
\hline Modelo & \multirow{2}{*}{ Estimador } & \multicolumn{5}{c}{ Parámetro estadístico } \\
& & GL & SC & CM & F & $P$ \\
\hline ANOVA & FN & 2 & 63 & 3.17 & 58.34 & $0.0001^{a}$ \\
& $\mathrm{CN}$ & 3 & 1.5 & 0.5 & 9.19 & $0.0001^{a}$ \\
& FN x CN & 6 & 2.2 & 0.37 & 6.75 & $0.0001^{a}$ \\
& Error & 273 & 14.82 & 0.05 & & \\
ANOVA & Tiempo & 2 & 7.23 & 3.63 & 57.05 & $0.0001^{a}$ \\
& Error & 282 & 17.96 & 0.06 & & \\
\hline
\end{tabular}

A mediano plazo, el crecimiento en longitud y número de hojas o raíces fue afectado por la fuente iónica de N (Tabla 2, Figura 2) y la interacción con la concentración de $\mathrm{N}$ influyó en el TR (Tabla 2, Figura 3). Las variaciones de mayor significancia de los iones $\mathrm{N}$ fueron registradas en LF y LH ${ }_{1}(p<0.0001)$. 

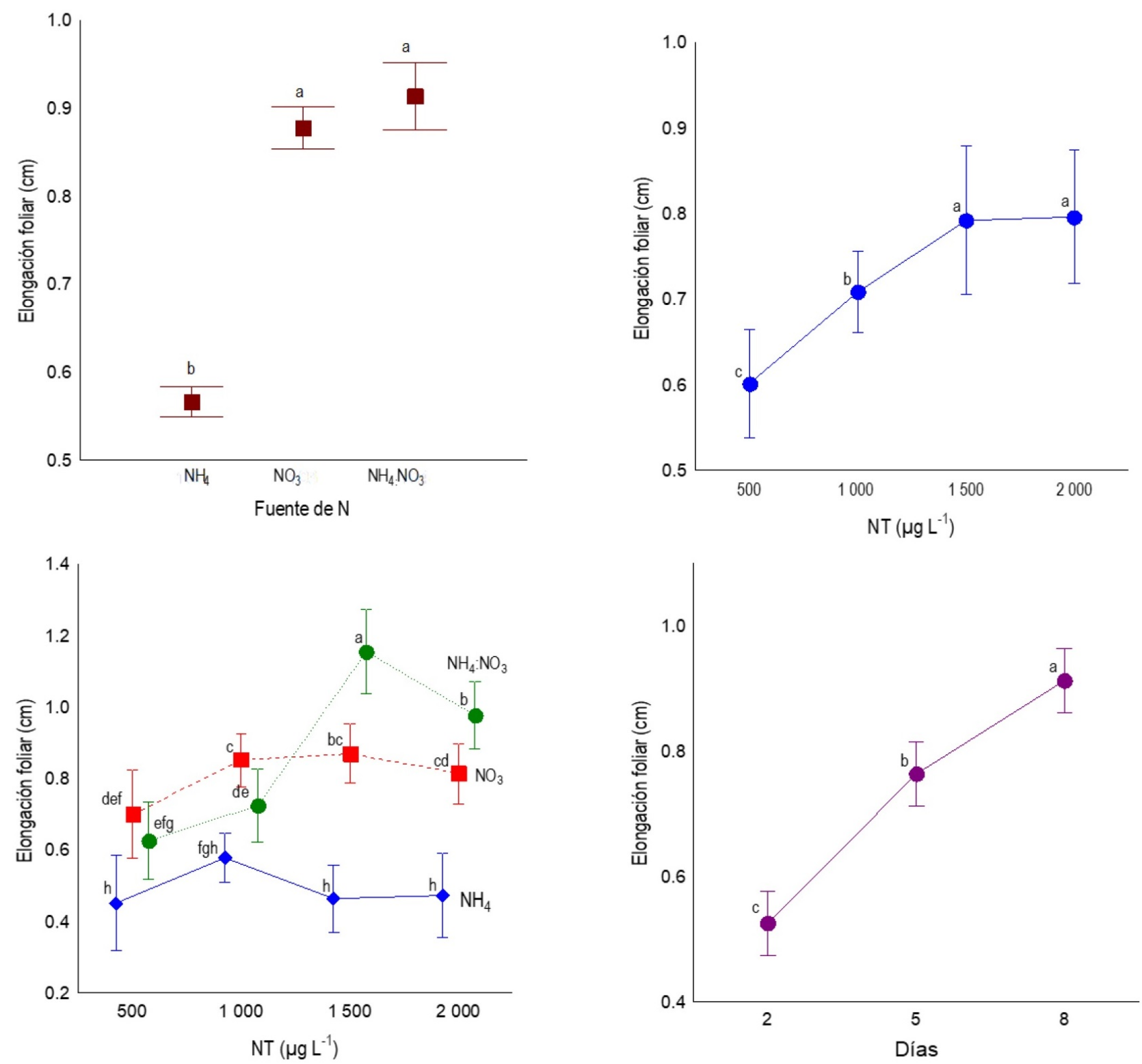

Figura 1. Evaluación a corto plazo: Elongación foliar de Vallisneria americana. a) Fuente de N, b) Concentración de $\mathrm{N}$, c) Fuente de $\mathrm{N} \times$ Concentración $\mathrm{N}$ y d) Tiempo de cultivo. Líneas verticales = promedios \pm error estándar. Promedios con literales distintas muestran diferencias significativas $(\mathrm{p}<0.05)$

El $\mathrm{NH}_{4}$, como fuente única de $\mathrm{N}$ en la columna de agua, produjo disminución en todos los parámetros de crecimiento de hojas y raíces; en cambio la fuente de $\mathrm{NH}_{4}: \mathrm{NO}_{3}$ benefició el crecimiento en magnitud similar a $\mathrm{NO}_{3}$, excepto en la $\mathrm{LH}_{2}$ y $\mathrm{NH}$. Únicamente, la $L R_{1}$ fue similar estadísticamente entre las fuentes de $\mathrm{N}(\mathrm{p}>0.05)$. El ión $\mathrm{NO}_{3}$ fue superior en el $90 \%$ de los crecimientos estimados. Por la interacción de la fuente de $\mathrm{N}$ y concentración de $\mathrm{N}$, el promedio de TR en $2000 \mu \mathrm{g} \mathrm{L}_{1}$ de $\mathrm{NH}_{4}$ fue el más bajo $(\mathrm{p}<0.05)$ y los promedios más altos coincidieron en 500 y 1500 $\mu \mathrm{g} \mathrm{L}_{1}$ de $\mathrm{NH}_{4}$ y las concentraciones de 500 a 2000 $\mu \mathrm{g} \mathrm{L}_{1}$ de $\mathrm{NO}_{3}$ y $\mathrm{NH}_{4}: \mathrm{NO}_{3}$. 
Tabla 2. Variación en el crecimiento de hojas y raíces de Vallisneria americana en la evaluación a mediano plazo. $\mathrm{FN}=$ Fuente de $\mathrm{N}, \mathrm{CN}=$ Concentración de $\mathrm{N}, \mathrm{G}=$ covariable $\left({ }^{c}=\mathrm{p}<0.05,{ }^{b}=\mathrm{p}<0.001,{ }^{a}=\mathrm{p}<0.0001\right)$.

\begin{tabular}{|c|c|c|c|c|c|c|c|}
\hline \multirow[t]{2}{*}{ Tejido } & \multirow{2}{*}{$\begin{array}{l}\text { Estimador } \\
\text { (cm o No). }\end{array}$} & \multirow[t]{2}{*}{ Factor(s) } & \multicolumn{5}{|c|}{ Parámetros estadísticos } \\
\hline & & & $\mathrm{GL}$ & SC & $\mathrm{CM}$ & $\mathrm{FoH}$ & $p$ \\
\hline \multirow[t]{14}{*}{ Hojas } & \multirow[t]{4}{*}{ LF } & FN & 2 & 0.48 & 0.24 & 12.36 & $0.0003^{b}$ \\
\hline & & $\mathrm{CN}$ & 3 & 0.08 & 0.03 & 1.40 & 0.271 \\
\hline & & $\mathrm{FN} \times \mathrm{CN}$ & 6 & 0.20 & 0.03 & 1.71 & 0.170 \\
\hline & & Error & 20 & 0.390 & 0.02 & & \\
\hline & \multirow{4}{*}{$\mathrm{LH}_{1}$} & FN & 2 & 2.37 & 1.19 & 23.25 & $0.0001^{a}$ \\
\hline & & $\mathrm{CN}$ & 3 & 0.38 & 0.13 & 2.51 & 0.087 \\
\hline & & $\mathrm{FN} \times \mathrm{CN}$ & 6 & 0.64 & 0.11 & 2.086 & 0.1 \\
\hline & & Error & & 1.02 & 0.05 & & \\
\hline & \multirow[t]{2}{*}{$\mathrm{LH}_{2}$} & FN & 2(32) & & & 6.65 & $0.036^{c}$ \\
\hline & & $\mathrm{CN}$ & $3(32)$ & & & 0.76 & 0.857 \\
\hline & \multirow[t]{4}{*}{$\mathrm{NH}$} & FN & 2 & 0.96 & 0.48 & 4.36 & $0.027^{c}$ \\
\hline & & $\mathrm{CN}$ & 3 & 0.16 & 0.05 & 0.47 & 0.704 \\
\hline & & $\mathrm{FN} \times \mathrm{CN}$ & 6 & 0.77 & 0.13 & 1.16 & 0.365 \\
\hline & & Error & 20 & 2.21 & 0.11 & & \\
\hline \multirow[t]{17}{*}{ Raíces } & \multirow[t]{4}{*}{ LR } & FN & 2 & 2.96 & 1.48 & 5.91 & $0.01^{c}$ \\
\hline & & $\mathrm{CN}$ & 3 & 0.79 & 0.26 & 1.05 & 0.392 \\
\hline & & $\mathrm{FN} \times \mathrm{CN}$ & 6 & 1.64 & 0.27 & 1.09 & 0.401 \\
\hline & & Error & 20 & 5.02 & 0.25 & & \\
\hline & \multirow[t]{5}{*}{$\mathrm{LR}_{1}$} & G & 1 & 1.70 & 1.70 & 5.34 & $0.01^{c}$ \\
\hline & & FN & 2 & 2.96 & 1.48 & 4.64 & 0.053 \\
\hline & & $\mathrm{CN}$ & 3 & 0.75 & 0.25 & 0.78 & 0.518 \\
\hline & & $\mathrm{FN} \times \mathrm{CN}$ & 6 & 1.03 & 0.17 & 0.54 & 0.773 \\
\hline & & Error & 19 & 6.06 & 0.32 & & \\
\hline & \multirow[t]{4}{*}{$\mathrm{LR}_{2}$} & FN & 2 & 3.30 & 1.65 & 7.55 & $0.004^{b}$ \\
\hline & & $\mathrm{CN}$ & 3 & 0.30 & 0.10 & 0.46 & 0.711 \\
\hline & & $\mathrm{FN} \times \mathrm{CN}$ & 6 & 2.16 & 0.36 & 1.65 & 0.186 \\
\hline & & Error & 20 & 4.36 & 0.22 & & \\
\hline & \multirow[t]{4}{*}{ TR } & FN & 2 & 0.73 & 0.37 & 10.79 & $0.0007^{b}$ \\
\hline & & $\mathrm{CN}$ & 3 & 0.07 & 0.02 & 0.71 & 0.557 \\
\hline & & $\mathrm{FN} \times \mathrm{CN}$ & 6 & 0.672 & 0.11 & 3.26 & $0.021^{c}$ \\
\hline & & Error & 20 & 0.681 & 0.03 & & \\
\hline
\end{tabular}

\section{DISCUSIÓN}

En la predicción in vitro, el ecotipo local de $V$. americana presentó variación en el crecimiento con los diferentes niveles de enriquecimiento de $\mathrm{NH}_{4}$, $\mathrm{NO}_{3}$ y $\mathrm{NH}_{4}: \mathrm{NO}_{3}$ sin manifestar síntomas de estrés tóxico en etapa juvenil. En el caso del incremento de $\mathrm{N}$ de $\mathrm{NH}_{4}$, la hipótesis se corroboró en el crecimiento de hojas y raíces con la inhibición de la elongación foliar y del brote completo. Estos resultados difieren con los hallazgos en campo de Cao et al. (2007), respecto a la mortalidad por estrés tóxico de $V$. natans por la amonificación en la columna de agua del sistema acuático, ya que, en el caso del sistema predictivo in vitro, las plantas de $V$. americana sobrevivieron frente al suministro de $\mathrm{NH}_{4}$. En cambio, los resultados de Li et al. (2008) y Zhao et al. (2016) con V. spi- nulosa y los experimentos de este estudio coincidieron con la ausencia de toxicidad bajo el enriquecimiento iónico de $\mathrm{NH}_{4}$ y de inhibición del crecimiento vegetativo con $\mathrm{NO}_{3}$. Las divergencias en las manifestaciones de estrés tóxico o fisiológico pueden ser atribuibles a los genotipos y a la condición ecológica general del ecosistema. El menor crecimiento foliar de $V$. americana en todas las concentraciones de $\mathrm{NH}_{4}$ confirma los efectos deletéreos del enriquecimiento de $\mathrm{N}$ en la columna de agua, pero los factores aditivos del sedimento pueden causar mayor impacto ( $\mathrm{Gu}$ et al. 2016). Como ejemplo, los procesos de amonificación y amoxidación en el sedimento, incluidas la nitrosoficación y nitrificación, se han vinculado al aumento dramático de la concentración $\mathrm{N}$ en el agua, convertido en amoníaco, que propicia la invasión exógena de la bacteria Escheri- 


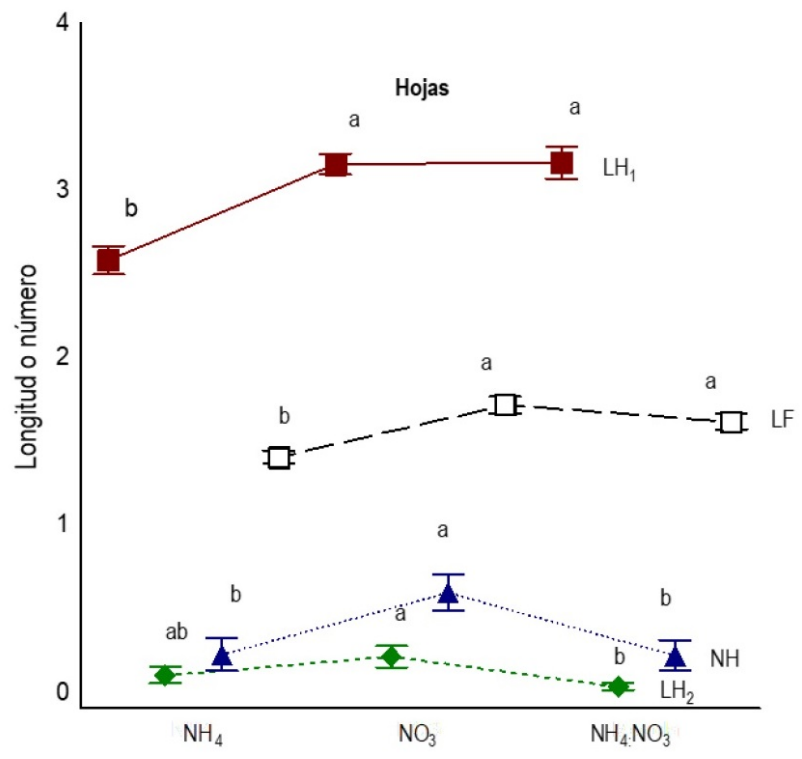

Fuente de $\mathrm{N}$

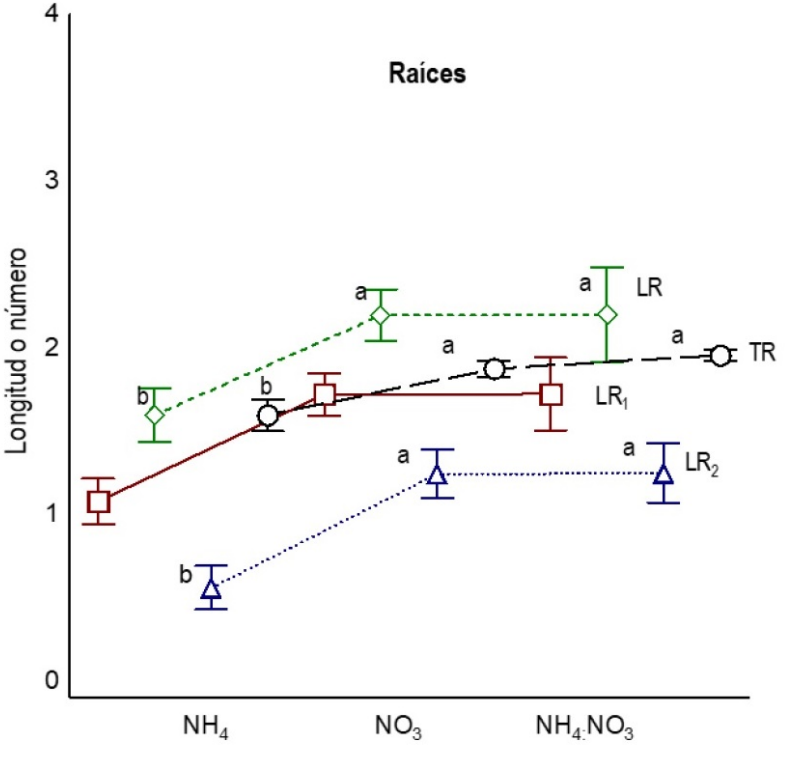

Fuente de $\mathrm{N}$

Figura 2. Evaluación a mediano plazo: Crecimiento de hojas y raíces de Vallisneria americana cultivada en ambientes enriquecidos con iones $\mathrm{NH}_{4}$, $\mathrm{NO}_{3}$ y $\mathrm{NH}_{4}: \mathrm{NO}_{3}$. Líneas verticales = promedios \pm error estándar. Promedios con literales distintas muestran diferencias significativas $(\mathrm{p}<0.05)$.

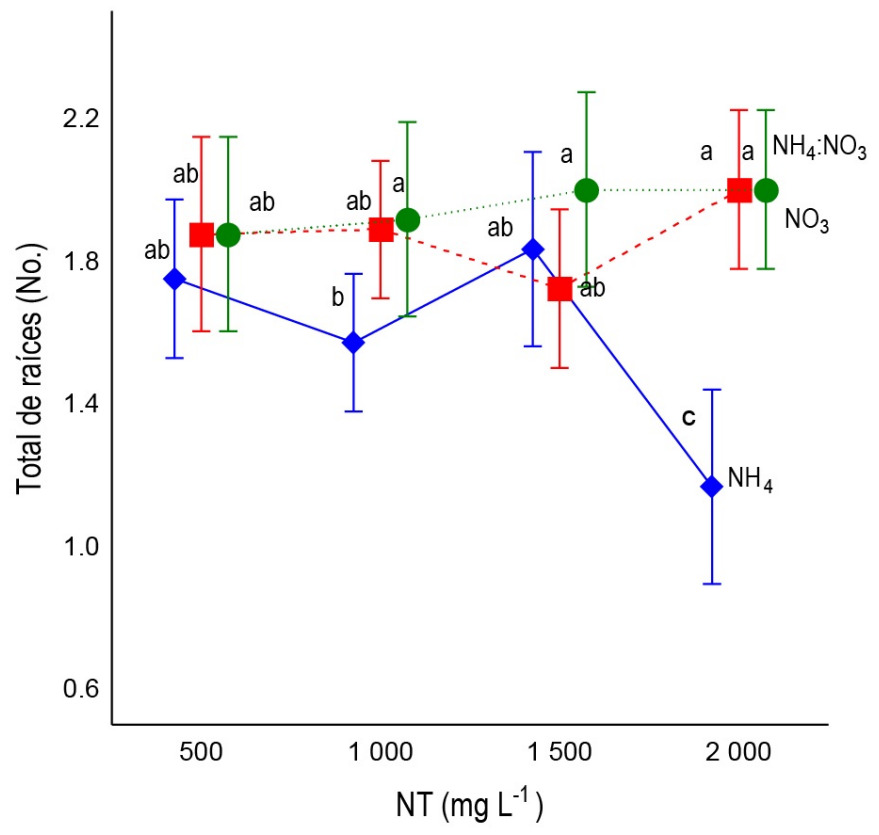

Figura 3. Evaluación a mediano plazo: Efecto combinado de la fuente de $\mathrm{N}$ y la concentración de $\mathrm{N}$ en el número de raíces totales de Vallisneria americana. Líneas verticales $=$ promedios \pm error estándar. Promedios con literales distintas muestran diferencias significativas $(p<0.05)$. 
chia coli en la vegetación sumergida de ecosistemas de agua dulce, así como el cambio significativo de la composición bacteriana en las biopelículas foliares y biopelículas de los sedimentos (Gu et al. 2020).

En el experimento de este estudio fue mayor el crecimiento de $V$. americana en concentración moderada de $\mathrm{NH}_{4}$ en combinación con una proporción baja de $\mathrm{NO}_{3}$. Este efecto positivo en la elongación foliar y en el número de raíces con suministro de $\mathrm{NH}_{4}: \mathrm{NO}_{3}$ fue pronunciado en concentraciones mayores de $\mathrm{N}$ externo y fue sobresaliente con suministro único de $\mathrm{NO}_{3}$. Lo que contradice el paradigma de la preferencia nutricional de las hidrófitas que reconoce al $\mathrm{NH}_{4}$ como la fuente principal de N (Cao et al. 2011, Piyanart et al. 2011). Aunque, las preferencias aparentes por fuentes de $\mathrm{N}$ resultan complejas (Hachiya y Sakakibara 2017). Por lo que el sistema in vitro y el aislamiento de factores ambientales interferentes pueden considerarse propicios para el crecimiento de $V$. americana con enriquecimiento de $\mathrm{N}$ en la columna de agua. La respuesta de crecimiento con suministro de $\mathrm{NH}_{4}: \mathrm{NO}_{3}$ se explica porque estas dos especies iónicas de $\mathrm{N}$ se forman durante el ciclo estacional del humedal (Kronzucker et al. 1999, Brix et al. 2002, Romero et al. 2006). Pero, la preferencia que manifiestan las especies vegetales por una fuente de $\mathrm{N}$ necesita interpretaciones más profundas en sistemas ecológicos como los humedales, debido a la variabilidad estructural y espacio-temporal inherente a los ecosistemas (Britto et al. 2013, Hachiya y Sakakibara 2017).

En las evaluaciones in vitro, las concentraciones de $\mathrm{NH}_{4}: \mathrm{NO}_{3}$ de máxima elongación foliar registradas se mantuvieron entre 1500 y $2000 \mu \mathrm{g}$ $\mathrm{L}^{-1}$, las cuales son equivalentes a $54 \mu \mathrm{M}(42 \mu \mathrm{M}$ de $\mathrm{NH}_{4}$ y $12 \mu \mathrm{M}$ de $\mathrm{NO}_{3}$ ) y $72 \mu \mathrm{M}\left(56 \mu \mathrm{M}\right.$ de $\mathrm{NH}_{4}$ y $16 \mu \mathrm{M}$ de $\mathrm{NO}_{3}$ ), respectivamente. Estas dos concentraciones de $\mathrm{NH}_{4}: \mathrm{NO}_{3}$ en magnitud micromolar contienen 3.5 veces más $\mathrm{NH}_{4}$ en relación al $\mathrm{NO}_{3}$, por lo que el resultado obtenido se puede explicar por las mayores tasas de adquisición de $\mathrm{N}$, debido a los iones $\mathrm{NH}_{4}$ y $\mathrm{NO}_{3}$ en las raíces de diversas especies acuáticas (Tylova-Munzarova et al. 2005). Mientras que en Oryza sativa fue sobresaliente la relación 3:1 de $\mathrm{NH}_{4}: \mathrm{NO}_{3}$ en función de la acumu- lación de $\mathrm{N}$ (Duan et al. 2007). En la misma especie, el suministro $100 \mu \mathrm{M}$ con una fuente dual de $\mathrm{N}$ fue sustancialmente mayor en la adquisición neta de $\mathrm{N}$ y la translocación a los brotes comparado con concentraciones equivalentes de $\mathrm{NO}_{3} \mathrm{O}^{\mathrm{NH}_{4}}$ individuales (Kronzucker et al. 1999). El máximo crecimiento y rendimiento obtenido con la combinación de estas dos fuentes de $\mathrm{N}$ ha sido atribuido a la desregulación de la entrada y metabolismo de $\mathrm{NH}_{4}$ por $\mathrm{NO}_{3}$ (Kronzucker et al. 1999, Duan et al. 2007, Hachiya y Sakakibara 2017). Algunos mecanismos moleculares que explican la naturaleza sinérgica de los iones $\mathrm{NH}_{4}$ y NO 3 en el crecimiento de las plantas fortalecen la interpretación del resultado obtenido con $V$. americana a corto y mediano plazo.

Por consiguiente, la elongación foliar a corto plazo y los marcadores de hojas y raíces a mediano plazo fueron predictores de estrés útiles para describir los impactos del enriquecimiento de $\mathrm{N}$ con fuentes de $\mathrm{N}$ oxidado y $\mathrm{N}$ reducido en el crecimiento de $V$. americana, tal como se reporta para monocotiledóneas terrestres (Bernstein et al. 1993, Rodríguez et al. 2004, Bultynck et al. 2004, Kavanová et al. 2006). La predicción reduccionista es útil para aplicaciones a estrés múltiple y como herramienta para regenerar o propagar angiospermas sumergidas encaminadas a acciones de repoblación y biomonitoreo del estado ecológico del ecosistema (Ailstock et al. 2010, Zarranz et al. 2010).

\section{CONCLUSIONES}

El crecimiento in vitro de plantas juveniles $V$. americana declinó principalmente por enriquecimiento de $\mathrm{NH}_{4}$, sin mostrar síntomas de estrés tóxico en las evaluaciones de corto y mediano plazo. Adicionalmente, se comprobó la hipótesis de inhibición en el crecimiento del brote completo con $\mathrm{NH}_{4}$ y en la regeneración de hojas con $\mathrm{NH}_{4}: \mathrm{NO}_{3}$, pero sin discriminar la influencia de la concentración de N. En contraste, la elongación foliar y el número de raíces fueron marcadores sensibles al incremento de $\mathrm{N}$, siendo menores en concentraciones altas de $\mathrm{NH}_{4}$ similares a las registradas en condiciones eutróficas en ecosistemas acuáticos de agua dulce. El 
conocimiento de estos efectos del enriquecimiento de $\mathrm{N}$ en la fisiología del crecimiento de $\mathrm{V}$. americana ofrece una base de información bioecológica para re- cuperar sus poblaciones a nivel global, en particular en la Reserva de la Biosfera Pantanos de Centla.

\section{LITERATURA CITADA}

Ailstock MS, Shafer D, Magoun AD (2010) Protocols for use of Potamogeton perfoliatus and Ruppia maritima seeds in large-scale restoration. Restoration Ecology 18: 560-573.

Balestri E, Cinelli F (2003) Sexual reproductive success in Posidonia oceanica. Aquatic Botany 75: 21-32.

Bautista-Regil J, Ruiz-Carrera V, Sánchez AJ, Salcedo-Meza AM, Florido R (2016) Stress to microscale of Vallisneria americana by enrichment of nutrients with stoichiometry N:P. Hidrobiológica 26: 53- 60.

Bernstein N, Läuchli A, Silk WK (1993). Kinematics and dynamics of sorghum (Sorghum bicolor L.) Leaf development at various Na/Ca salinities: I. Elongation growth. Plant Physiology 103:1107-1114.

Brix H, Kirsten D,Lorenzen B (2002) Root-zone acidity and nitrogen source affects Typha latifolia L. growth and uptake kinetics of ammonium and nitrate. Journal of Experimental Botany 53: 2441-2450.

Britto DT, Kronzucker HJ (2013) Ecological significance and complexity of N-source preference in plants. Annals of Botany 112: 957-963.

Bultynck L, ter Steege MW, Schortemeyer M, Poot P, Lambers H (2004) From individual leaf elongation to whole shoot leaf area expansion; a comparison of three Aegilops and two Triticum species. Annals of Botany 94: 99-108.

Cao T, Xie P, Ni L, Wu A, Zhang M, Wu S, Smolders AJP (2007) The role of NH4+ toxicity in the decline of the submersed macrophyte Vallisneria natans in lakes of the Yangtze River basin, China. Marine and Freshwater Research 58: 581-587.

Cao T, Xie P, Li Z, Ni L, Zhang M, Xu J (2009) Physiological stress of high $\mathrm{NH}_{4}^{+}$concentration in water column on the submersed macrophyte Vallisneria natans L. Bulletin of Environmental Contamination and Toxicology 82: $296-9$

Cao TE, Ni L, Xie P, Xu J, Zhang M (2011) Effects of moderate ammonium enrichment on three submersed macrophytes under contrasting light availability. Freshwater Biology 56: 1620-1629.

Cordovil CMDS, Cruz S, Brito AG, Cameira MDR, Poulsen JR, Thodsen H, Kronvang B (2018) A simplified nitrogen assessment in Tagus River Basin: A management focused review. Water 10: 406. Doi: 10.3390/w1004 0406.

Cruz-Ramírez AK, Salcedo MA, Sánchez AJ, Barba E, Mendoza JD (2019) Relationship among physicochemical conditions, chlorophyll-a concentration, and water level in a tropical river-floodplain system. International Journal of Environmental Science and Technology 16: 3869-3876.

Duan HY, Zhang LY, Ye TL, Fan RX, Xu HG, Shen RQ (2007) Responses of rice cultivars with different nitrogen use efficiency to partial nitrate nutrition. Annals of Botany 99: 1153-1160.

Eaton AD, Clesceri LS, Greenberg AE (2000) Standard methods for the examination of water and wastewater. 19th Ed. American Public Health Associaton (APHA). Maryland. Joint Editorial Board. Washington D.C. $139 p$.

Gao J, Ren P, Zhou Q, Zhang J (2019) Comparative studies of the response of sensitive and tolerant submerged macrophytes to high ammonium concentration stress. Aquatic Toxicology 211: 57-65. 
Gu J, Xu Z, Jin H, Ning X, He H, Yu J, Jeppesen E, Li K (2016) Response of Vallisneria natans to increasing nitrogen loading depends on sediment nutrient characteristics. Water 8: 563. Doi: 10.3390/w8120563.

Gu L, Wu J, Hua Z, Chu K (2020) The response of nitrogen cycling and bacterial communities to E. coli invasion in aquatic environments with submerged vegetation. Journal Environmental Management 261, 110204. Doi: 10.1016/j.jenvman.2020.110204.

Hachiya T, Sakakibara H (2017). Interactions between nitrate and ammonium in their uptake, allocation, assimilation, and signaling in plants. Journal of Experimental Botany 68: 2501-2512.

Johnson AJ, Orth RJ, Moore KA (2019) Herbivory regulates the establishment of a native species of submerged aquatic vegetation (SAV) in a tidal estuary of the USA. Oecologia 190: 639-650.

Kavanová M, Lattanzi FA, Grimoldi AA, Schnyder H (2006) Phosphorus deficiency decreases cell division and elongation in grass leaves. Plant Physiology 141: 766-775.

Kouamé MK, Dietoa MY, Edia EO, Da Costa SK, Ouattara A, Gouréne G (2011) Macroinvertebrate comunities associated with macrophyte habitats in a tropical man-made lake (Lake Taabo, Côte d'Ivoire). Knowledge and Management of Aquatic Ecosystems 400: 1-18.

Kronzucker HJ, Siddiqi MY, Glass ADM, Kirk GJD (1999) Nitrate-ammonium synergism in rice: A subcellular analysis. Plant Physiology 119: 1041-1046.

Le Moal M, Gascuel-Odoux C, Ménesguen A, Souchon Y, Étrillard C, Levain A, Pinay G (2019) Eutrophication: a new wine in an old bottle? Scince of the Total Environment 651: 1-11. Doi: 10.1016/j.scitotenv.2018.09.139.

Leoni V, Pasqualini V, Pergent-Martini C, Vela A, Pergent G (2008) Morphological responses of Posidonia oceanica to experimental nutrient enrichment of the canopy water. Journal of Experimental Marine Biology and Ecology 339: 1-14.

Li W, Zhang Z, Jeppesen E (2008) The response of Vallisneria spinulosa (Hydrocharitaceae) to different loadings of ammonia and nitrate at moderate phosphorus concentration: a mesocosm approach. Freshwater Biology 53: 2321-2330.

Li Y, He Q Ma X, Wang H, Liu C, Yu D (2019) Plant traits interacting with sediment properties regulate sediment microbial composition under different aquatic DIC levels caused by rising atmospheric CO2. Plant and Soil 445: 497-512.

Lone PA, Bhardwaj AK, Shah KW (2014) Macrophytes as powerful natural tools for water quality improvement. Research Journal of Botany 9: 24-30.

McFarland D (2006) Reproductive ecology of Vallisneria americana Michaux. SAV Technical Notes Co-llection (ERDC/TNSAV-06-04). Vicksburg,MS: U.S. Army Engineer Research and Development Center. EE.UU. $27 p$.

Moreno-Marín F, Brun FG, Pedersen MF (2018) Additive response to multiple environmental stressors in the seagrass Zostera marina L. Limnology and Oceanography 63: 1528-1544.

Naiman RJ, Dudgeon D (2011) Global alteration of freshwaters: influences on human and environmental wellbeing. Ecological Research 26: 865-873

Padgett EP, Leonard TR (1993) Contamination of ammonium-based solutions by nitrifying organisms and the conversion of ammonium to nitrate. Plant Physiology 307: 127-135.

Piyanart S, Prasit W, Arunothai J (2011) Growth, morphology, ammonium uptake and nutrient allocation of Myriophyllum brasiliense Cambess, under high $\mathrm{NH}_{4}^{+}$concentrations. Ecotoxicology 20: 2011-2018. 
Rodríguez AA, Córdoba RA, Ortega L, Taleisnik E (2004) Decreased reactive oxygen species concentration in the elongation zone contributes to the reduction in maize leaf growth under salinity. Journal of Experimental Botany 55: 1383-1390.

Romero J, Lee KS, Pérez M, Mateo MA, Alcoverro T (2006) Nutrient dinamic in seagrass ecosistems. In: Larkum AWD, Orth RJ, Duarte C (eds) Biology, Ecology and Conservation Seagrasses. Springer Netherlands. Holland. pp: 159-192.

Ruiz-Carrera V, Sánchez AJ (2008) Development of a model for in vitro culture of Vallisneria americana Michx. Universidad y Ciencia 24: 205-218.

Salcedo MA, AJ Sánchez, de la Lanza G, Kampichler C, R Florido (2012) Condición ecológica del humedal tropical Pantanos de Centla. In: Sánchez AJ, Chiappa-Carrara X, Brito PR (eds) Recursos Acuáticos Costeros del Sureste Volumen II. CONCYTEY. Mérida. México. 1096p.

Salk KR, Bullerjahn GS, McKay RML, Chaffin JD, Ostrom NE (2018) Nitrogen cycling in Sandusky, Bay Lake Erie: oscillations between strong and weak export and implications for harmful algal blooms. Biogeosciences 15: 2891-2907.

Sánchez AJ, Florido R, Salcedo MA, Ruiz-Carrera V, Montalvo-Urgel H. Raz-Guzman A (2012) Macrofaunistic diversity in Vallisneria americana Michx. in a tropical wetland, Southern Gulf of Mexico. In: Mahamane A (ed) Ecosystems I. InTech. Rijeka, Croatia. pp: 1-26.

Schloesser DW, Manny BA (2007) Restoration of Wildcelery, Vallisneria americana Michx., in the Lower Detroit River of the Lake Huron-Lake Erie Corridor. Journal of Great Lakes Research 33: 8-19.

Teh CY, Mahmood M, Shaharuddin NA, Ho CL (2014) In vitro rice shoot apices as simple model to study the effect of $\mathrm{NaCl}$ and the potential of exogenous proline and glutathione in mitigating salinity stress. Plant Growth Regulation 75: 771-781.

Tootoonchi M, Gettys LA, Thayer KL, Markovich IJ, Sigmon JW, Sadeghibaniani S (2020) Ecotypes of aquatic plant Vallisneria americana tolerate different salinity concentrations. Diversity 12: 65. Doi: 10.3390/d120200 65.

Tylova-Munzarova E, Lorenzen B, Brix $\mathrm{H}$, Votrubova $\mathrm{O}$ (2005) The effects of $\mathrm{NH}_{4}^{+}$and $\mathrm{NO}_{3}^{-}$on growth, resource allocation and nitrogen uptake kinetics of Phragmites australis and Glyceria maxima. Aquatic Botany 81: 326-342.

Yang X, Wu X. Hao H, He Z (2008) Mechanisms and assessment of water eutrophication. Journal of Zhejiang University Science B 9: 197-209.

Zaman T, Asaeda $\mathrm{T}$ (2013) Effects of $\mathrm{NH}_{4}-\mathrm{N}$ concentrations and gradient redox level on growth and allied biochemical parameters of Elodea nuttallii (Planch.). Flora-Morphology, Distribution, Functional Ecology of Plants. 208: 211-219.

Zarranz ME, González-Henríquez N, García-Jiménez P, Robaina RR (2010) Restoration of Cymodocea nodosa seagrass meadows through seed propagation: germination in vitro, seedling culture and field transplants. Botanica Marina 53: 173-181.

Zhao S, Yin L, Chang F, Olsen S, Søndergaard M, Jeppesen E, Li W (2016) Response of Vallisneria spinulosa (Hydrocharitaceae) to contrasting nitrogen loadings in controlled lake mesocosms. Hydrobiologia 766: 215223. 